tion does not affect the neuritis and loss of sight is in the membranes of the brain and cord." Atrophy probably due to neuritis as vision is rarely affected of the optic nerve in this disease may be distinguished by the abscess itself.

Multiple sclerosis occurs at all ages but more frequently in the first half of adult life, between the ages of 20 and 30 years. It may affect any portion of the brain, hence the symptoms vary. The cranial nerves are often affected, especially the first, second, third, fifth and seventh. Loss of muscular control is a common and early symptom, movements are peculiar and jerky but cease when the parts are at rest. Nystagmus is very common. The "eye symptoms are frequent and important. The optic nerve may suffer in various ways. There may be impairment of sight in one eye or both, often with an irregular contraction in the field of vision, and without at first any visible changes in the optic nerve to account for it. This condition depends upon the development of an islet of sclerosis in one or both optic nerves, or in the chiasma. It may progress almost to complete blindness of one eye. After a time secondary atrophy supervenes, and can be seen with the ophthalmoscope; occasionally the atrophy is preceded by slight neuritis, when sclerosis is near the eye ball. In some cases again there is a primary atrophy of the optic nerves, exactly like that which occurs in tabes, with a similar failure of sight, proportioned to the visible alteration in the nerve. Both eyes usually suffer, but one is often affected earlier than the other. This complication is, according to my own experience, rather more frequent than the form first described, and is of much pathologic interest." There is at times loss of accommodation, less frequently light reflex and the pupils may be unequally dilated.

Bulbar paralysis or labio-glosso-pharyngeal paralysis occurs generally in the second half of life and in males oftener than females. The causes are obscure; sometimes mental depression and anxiety, exposure to cold, debilitating influences and injuries, as blows upon the back of the neck. Optic nerve atrophy is of rare occurrence in this disease and of course when it occurs the symptoms of the disease would be a guide in diagnosis.

Paralysis of the insane, general paralysis.-Vision is frequently affected by this disease, the ophthalmoscope showing choked disk or consecutive atrophy according to the stage of the affection. General paralysis is a disease of the well-to-do, the rich rather than the poor, and occurs generally among those who live a busy, hurrying life rather than among those who reside in rural districts and lead quiet lives. It is often seen in the highly educated, in males oftener than females and about the prime of life. Hot climates, certain pursuits and excessive sexual indulgence are known to be exciting causes. Child-bearing and over indulgence in alcoholic liquors may also be a cause. It frequently follows or is associated with locomotor ataxia. The symptoms of this disease are so well known that it should not be difficult to diagnose the cause of the optic nerve atrophy. In addition to the general symptoms there may be at times dilatation of the pupils, myosis usually occurring at an early stage, but a difference in the size of the pupils is still more definite. The "Argyll-Robertson pupil" is often seen; these symptoms usually occur in other diseases of the brain and are chiefly valuable as indicating central disease. The cause of this disease is "a progressive degeneration of the encephalo-spinal nervous system attended by subacute inflammatory changes from that eaused by tumor of the brain, syphilis of the brain, etc., by the absence of the symptoms that indicate those lesions and especially from the latter by the failure to get improvement by the use of mercury and iodid of potassium. Treatment is of no avail in this form of atrophy.

Brchin tumors cause diffused symptoms, headache, vertigo, vomiting, general epileptiform convulsions, apoplectiform attacks, psychic disturbances and choked disk. Choked disk is found more frequently ( 54 per cent.) in cases when the tumor involves the corpora quadrigemina. Direct pressure on the optic tract causes atrophy, at times, without choked disk. Tremors and localized spasms are valuable diagnostic signs, because, first, they are more frequent with tumors than other lesions; and second, they are more frequent in the cortex; third, more frequent in the motor zones. In more than one-third of all cases localized spasmus and contractions exist at some time during the disease and when present they indicate localization in the cortex oftener than elsewhere, next the corpus striatum and optic thalamus. Paralysis is gradual and distinguishes it from hemorrhage. Tumors of the corpora striata, corpora quadrigemina and lenticular nuclei cause hemiplegias more slowly than hemorrhage. Disturbances of vision are very frequent as symptoms of brain tumor: 1, atrophy of optic nerve following choked disk; 2, deviation of the eye ball or eye lids from isolated or combined paralysis of the nerves supplying the ocalar muscles, the third, sixth and seventh; 3, amblyopia, or amaurcsis, resulting from direct affection of the optic nerve in its course through the cranium, or at its cerebral centers, the mode of development being, therefore, almost precisely analogous to that of paralysis of any of the nervous tracts by direct compression. The optic chiasma or nerve is liable to pressure from tumors arising from the base of the cranium or from the hypophysis, and also from the peduncles. Hemiopia is produced when one tract or one side of the chiasma is compressed. Tumors of the thalamus do not often cause blindness. Tumors of the corpora quadrigemina have a much larger percentage of blindness than those of any other part and those of the cerebellum come next.

\title{
THE DIFFERENTIAL DIAGNOSIS BETWEEN SIMPLE GLAUCOMA AND OPTIC NERVE ATROPHY.
}

Read in the Section on Ophthalmology at the Forty-seventh Anuual Meeting of the American Medical Association at Atlanta. Georgia, May 5-8, 1896.

BY B. ALEXANDER RANDALL, M.D.

Ophthalmic and Aural Surgeon to the Methodist and the Children's Hospitals, etc. Piilladelphi, Pa

At first thought little difficulty would seem to present itself in differentiating simple glaucoma from optic atrophy, and in the majority of cases any careful student ought to succeed. A table can easily be constructed, giving the typical like and unlike symptoms with such clearness of "deadly parallel columns" as to make the tyro sure that only a blockhead could err. Yet some or all of our differentiating signs are at times lacking or doubtful, anomalous or casual complications are encountered, and experience teaches that the best-posted observers are tried at 
times to the uttermost by the uncertainties of some cases. And when a case passes from one to another with varying diagnosis, the later student will do well to temper his self-gratulations by remembering that the cardinal symptoms may have been wholly masked before, and better fortune rather than superior skill has given him the advantage.

It behooves us, therefore, to study out, with all care, every detail, even in cases that appear perfectly clear; for most of us have had patients in whom at one time no trace of glaucoma has been detected, while shortly afterward they have presented themselves with so marked a stage of the affection as to convince us that exhaustive study ought earlier to have recognized it. I shall not soon forget two ladies sent me, about the same time, by one physician, each of whom had full development of glaucoma in the left eye when they returned two years later. One had rainbow vision, that made my study exhaustive, though vain for other symptoms; the other had no suggestions of it. Both were irretrievably neglected, when seen again. Such cases passing into other hands must arouse doubts of the competency of the earlier adviser, unless there is full and frank communication with him. On the other hand, optic atrophy may strongly suggest glaucoma and lead to a tentative or positive suggestion of iridectomy which the subsequent history shows to have been groundless.

In simple glaucoma and optic atrophy we may usually find the contrasted symptoms of the following table; but it must never be forgotten that they may be wholly absent, or obscured if present.

\section{TABULATION OF SYMPTOMS.}

Simple Glaucoma

Tension increased.

Cornea hazy and anesthetic.

Anterior chamber shallow or obliterated.

Iris atrophic and discolored.

Pupil dilated and sluggish or fixed.

Reflex yellowish-green, "glaucous."

Perforating vessels dilated and tortuous.

History of pain and obscurations.

Unilateral character usual at first.

Limitation of field usually to nasal side.

Color-fields commensurate to formfields.

Rainbow - vision about lights.

Knee-jerks unaffected.

Optic disc cupped, margins overhang ing.

Halo of choroidal atrophy usual.

Nerve-tissue greenish in shadow.

Arteries and veins pulsating.

he cardinal symptom of increased tension is variable and often uncertain. Harlly any two observers will exactly agree as to the tension of an eye, and a rigid sclera, a tense eyelid or a rude touch may easily cause error. Palpation had better be done with the index fingers of both hands applied to one eye at a time and then transferred to the other for comparison. In doubtful cases it may be done upon the uncovered sclera, but it is usually better to have the patient look very far down and palpate above the tarsal cartilage. If there are intermissions in the symptoms, we may expect to find corresponding falls of the tension to the normal. With a shrinking patient the surgeon must be gentle and not over-endowed with thumbs. The superficial corneal haze, which gives it a slight "pin-stuck" appearance like ground-glass or a mirror that has been breathed upon, may be a very delicate change and, like the anesthesia, demand close observation to detect it. It is doubtless due to edema of the epithelium and pressure upon the nerves. 'Though most marked near the pole, it is nearly uniform, in contrast to more localized keratitic changes. Individual differences in the depth of the anterior chamber, with a tendency to shallow with advance of life, must be taken into account; and the pressing forward of the lens is not usually very marked, but the appearance of the iris is usually quite characteristic. Altered color, vague detail of the surface or irregularities of structure and a general suggestion of atrophy are rarely lacking, and can easily raise a question as to iritis present or past, by the peculiarity of some of the changes. So too the pupil, semi-dilated and fixed as a rule, oval oftener than round, at best sluggish and imperfect in action - it is generally more abnormal in even the beginning of glaucoma than it becomes in any but the last stages of optic atrophy. The peculiarities of action to accommodative stimulus but not to light, which Argyll-Robertson pointed out; the hemianopic reflex of Wernicke; the consensual action of an iris otherwise immobile, are rare in any glaucomatous phase, and may be determinate of the character of the nerve degeneration which we generally have to diagnosticate largely on negative data, by exclusion. The yellowish-green reflex, often seen in the pupil of the glaucomatous eye, may have some counterpart in the opalescent haze of the lens in the patient with atrophic nerves, who is usually well advanced in life and has more or less arcus senilis and other diminutions of the transparency of his media. Yet it joins in the formation of the external picture of glaucoma which the skilled observer can often recognize at a glance, although analysis of its details shows each of them so vague as to bring doubt as to its real existence. Rarely lacking, however, to complete the picture and demonstrate the congestion of the ciliary vessels is the turgid, dark fullness of the perforating veins as they emerge from the sclera, several millimeters back from the cornea, and meander beneath the conjunctiva. Many conditions of ciliary congestion are marked by the undue prominence of these vessels; but in none of the active conditions are they as marked as they usually are in simple glaucoma. Other injection of the anterior segment, such as may be great in the acute and subacute glaucomatous attacks, may be wholly lacking in all phases of simple glaucoma, and yet the observer will hardly fail to receive an impression of congestion, out of proportion to the expanse of these vessels.

The history ought to help the diagnosis greatly, by the statement of pain in the eye, hemicrania or "neuralgia," coincident with the periods of obscuration and heralded by rainbows around the light. Both affections are likely to be bilateral, but in optic atrophy the second eye follows but little behind the other in its decline; whereas years may intervene before the glaucoma that has blinded one eye manifests itself in the other.

Mensuration of the visual field should never be neglected, and although its showings are never to be accepted as pathognomonic, much can generally be learned from the charts thus obtained. Color-vision is generally much lowered in optic atrophy, red and green even wholly lost or presenting very restricted areas of perception, with scotoma regions, where the recognition is weak, if not quite lost. The form-tield 
is more commonly restricted uniformly in the spinal and some of the cerebral cases, although hemianopsia and quadrant defects are not unusual, and may be very helpful in localization of the underlying lesions. In glaucoma the general form-field suffers proportionately more and may be narrowed down, more especially on the nasal side, until mere central vision remains, and the patient "sees as through a gun barrel."

The knee jerks and other tendon and muscle reflexes are well worthy of careful study in a number of our eye cases, but especially in any where plantar numbness, lancinating pains in the extremities and imperfect station or coördination suggest spinal disease. None of these defects are to be expected in glaucomatous conditions, although it is not impossible to have glaucoma attack an individual giving such evidences of lesion of the cerebro-spinal axis.

It is to the ophthalmoscope especially that we turn, however, for our diagnosis in many of these uncertain cases. Here the picture may be unmistakable, when much was doubtful in all other directions. Yet we can be over-confident as to its help, and find a picture that is almost typical of glaucoma when none is present, or that gives no confirmation to a diagnosis that is unmistakable in spite of such negative findings. It is easy to make a set of diagrams, as does Fuchs, to define the difference between physiologic, atrophic and pressure excavation of the nerve head; but in fact we may meet combinations or transpositions of such appearances. Jaeger's admirable atlas gives us some of the puzzling findings, while others have probably never been reported. The total cup, with overhanging edge and greenish shadow, may seem typically present in cases with normal fields and perfect central vision; and we look again in a vain effort to see where healthy nerve fibers can find a place at the margin of such a disc. So, on the contrary, we may find not only acute, but chronic glaucomas, in which neither the ophthalmoscope nor even anatomic study later, under the microscope, can demonstrate superficial cupping nor underlying depression of the lamina cribrosa. Most trying is such an inconclusive or contradictory finding in such cases where one eye has already been lost by unmistakable glaucoma, and the other is affected, but with a condition too little defined to make us positive in deciding either for or against iridectomy.

NoTE.--Numerous original illustrations of the typical and the contradictory appearances of eye grounds, fields and anatomic sections were thrown upon the screen with the stereopticon.

\section{SPONGE GRAFTING IN THE ORBIT FOR SUPPORT OF ARTIFICIAL EYE.}

Read in the Section on Ophthalmology, at the Forty-seventh Aunual Meeting of the Atlanta, Ga., May 5-8, 189

BY E. OLIVER BELT, M.D.

WASHINGTON, D. C.

PROFFSSOR OF OPHTIIALMOLOGY AND OTOLOGY, HOWARD UNIVERSITY, AND OCULIS'T AND AURIST TO FRENDMEN'S HOSPITAL.

To a skilled oculist the ordinary enucleation of an eyeball is one of the simplest operations he is called upon to perform, and yet there are some cases in which so much of the conjunctiva is destroyed by careless or ignorant operators, that it is impossible for an artificial eye to be worn afterward. This is very annoying to a patient, and we can not blame him if he never forgives the doctor who has so afflicted him.
Such cases emphasize the fact that it is the duty of the surgeon not only to operate skillfully for the immediate relief desired, but he should do everything in his power to prevent disfigurement, and when an organ or limb can not be restored to usefulness, he should at least obtain the best cosmetic results possible. After an eyeball has been removed and an artificial eye inserted, very frequently it is not as prominent as the natural eye. It has a sunken appearance which attracts attention, at once, to the fact that the eye is artificial. To remedy this defect has been a problem for ophthalmologists for years.

One of the most satisfactory operations heretofore practiced is that known as Mule's operation, which consists in the evisceration of the contents of the eye, and the insertion of a hollow glass globe in the sclerotic. This is sewed in, and proves fairly satisfactory, but there is some danger of the globe being broken, and occasionally the stitches come out and the globe escapes. There is also the risk of sympathetic ophthalmia, where the entire eyeball is not removed. To obviate these difficulties and at the same time secure a full orbit, I have devised a method of sponge grafting which seems to meet the requirements without the disadvantages of other methods.

The operation is a simple one and is performed as follows: The eyeball is removed by the ordinary method under strict asepsis. After all hemorrhage is arrested the socket is washed out with a formalin solution, 1 to 1,000 , followed by sterilized salt solution. A globe of fine, soft sponge, about three-fourths the size of the eyeball, previously sterilized in 5 per cent. formalin solution and rinsed in the salt solution, is then inserted into the socket, or capsule of Tenon. The conjunctiva is brought together and sewed with rat-tail sutures. The eyelids are then closed with compress and bandage. In a few weeks the sponge is filled with new tissue, which in time becomes firm, solid tlesh, making a full orbit and a fine support for the artificial eye. The sponge fibers are apparently absorbed.

I have performed this operation in five cases with fairly good results in all, but union of the conjunctiva has not been firm enough to prevent some of the stitches from breaking or cutting out and the wound gaping. In future cases, to relieve this strain on the conjunctiva and to obtain good motion of the eye, I think of uniting the opposing recti muscles with rattail sutures, and then the conjunctiva over that by the purse-string.or subcutaneous suture. By this method we should get union by first intention, good motion of the stump and a full orbit. There seems to be no danger from infection of the sponge, for in two cases the eye was removed for panophthalmitis. Not only has this operation advantages over other methods in recent cases, but in old cases in which the eye was removed months or years ago, where there is this sunken appearance of the artificial eye, the socket might be reopened and a sponge inserted.

Since making my investigation, I find that Prof. D. J. Hamilton of Edinburgh practiced sponge grafting for old ulcers in 1880, since which time a few other physicians have tried it in fresh wounds and some other similar conditions that skin grafting has been used in. However, I can not find in all the medical literature in the library of the Surgeon-General's office any mention of sponge being used as I bave suggested and tried.

Case 1.-E. I., colored, aged about 30, came to Freedman's 